\title{
Molecular Alterations of $h$-warts/LATS1 Tumor Suppressor in Human Soft Tissue Sarcoma
}

\author{
Masanori Hisaoka, Atsuko Tanaka, and Hiroshi Hashimoto \\ Department of Pathology and Oncology, School of Medicine, University of Occupational and Environmental Health, \\ Kitakyushu, Japan
}

\begin{abstract}
SUMMARY: $h$-warts/LATS1 is a human homolog of the Drosophila warts tumor suppressor gene, which functions as a component of the mitotic apparatus. LATS1-deficient (Lats $1^{-1-}$ ) mice have been reported to develop ovarian stromal tumors and soft tissue sarcomas. We investigated the status of the $h$-warts/LATS1 in 50 human soft tissue sarcomas to address its potential function as a tumor suppressor in human sarcoma development. In our RT-PCR assay, 7 (14\%) (3 of 4 myxoid liposarcomas, 3 of 7 leiomyosarcomas, 1 of 9 malignant fibrous histiocytomas) of 50 sarcomas examined had no or a reduced expression of the $h$-warts/LATS1, indicating down-regulated gene expression. We further analyzed alterations of the $h$-warts/LATS1 in these seven sarcomas. Using microsatellite markers for chromosome 6q23-25.1, to which the $h$-warts/LATS1 is localized, an allelic loss of this locus was detected in one leiomyosarcoma, in which a missense point mutation of the $h$-warts/LATS1 was detected by PCR single-strand conformation polymorphism. Clusters of $\mathrm{CpG}$ dinucleotides in a 5' putative promoter region were hypermethylated in the other six sarcomas. Our data suggest that the molecular alterations of the $h$-warts/LATS1 could be of pathologic importance in human sarcomagenesis. (Lab Invest 2002, 82:1427-1435).
\end{abstract}

$T$ umor suppressor genes are those that, when functioning normally, prevent the development of tumors; losses of their normal functions (inactivation) are required for tumorigenesis. Allelic losses, gene mutations, and cryptic deletions are often detected as mechanisms involved in the inactivation of tumor suppressor genes. In addition, hypermethylation of promoter regions of genes is considered another important mechanism for down-regulation or "silencing" of the genes. Tumor suppressor genes can be subdivided into two groups: genes that directly regulate tumor growth by inhibiting cell proliferation or promoting cell death ("gatekeepers") and genes whose inactivation causes genetic instability, which in turn leads to mutations that promote tumor growth ("caretakers") (Kinzler and Vogelstein, 1998). p53, RB-1, WT1, NF1, APC, VHL, BRCA1, BRCA2, E-CAD, $p 16^{I N K 4 A} / p 14^{A R F}$, and $p 15^{I N K 4 B}$ genes are now included as members in the growing list of human tumor suppressor genes (Hooper, 1998).

The warts (also referred to as LATS) was identified based on its ability to act as a tumor suppressor gene in Drosophila. A human homolog of this gene, $h$-warts/ LATS1, has recently been isolated (Nishiyama et al,

DOI: 10.1097/01.LAB.0000032381.68634.CA

Received July 2, 2002.

This work was supported in part by a 2000 Grants-in-Aid for Scientific Research (C) (No. 12670184) and Encouragement of Young Scientists (No. 12770102) from the Japan Society for the Promotion of Science and by a grant for cancer research from the Fukuoka Cancer Society, Japan. Address reprint requests to: Dr. Hiroshi Hashimoto, Department of Pathology and Oncology, School of Medicine, University of Occupational and Environmental Health, 1-1 Iseigaoka, Yahatanishi-ku, Kitakyushu 8078555, Japan.E-mail: hiroshi@med.uoeh-u.ac.jp
1999; St John et al, 1999; Tao et al, 1999). h-warts/ LATS1 encodes a putative serine/threonine kinase, which is phosphorylated in a cell cycle-dependent manner and forms a complex with CDC2 in early mitosis, suggesting its function as a negative regulator of CDC2/cyclin complexes during cell division (Hirota et al, 2000; Nishiyama et al, 1999; Tao et al, 1999). Notably, $h$-warts/LATS1-deficient (Lats $1^{-/-}$) mice spontaneously develop ovarian stromal tumors and soft tissue sarcomas and have a significant susceptibility to induction of the latter tumors (St John et al, 1999). Except for p53-deficient or $p 16^{I N K 4 A}$-deficient mice (Serrano et al, 1996; Williams and Jacks, 1996), few animal models lacking tumor suppressor genes have been reported to be sensitive to sarcoma development. Besides, the status of the $h$-warts/LATS1 in human neoplasms has not been investigated, except for one recent study on gene mutations in human breast cancer (Morinaga et al, 2000).

In this study, the role of $h$-warts/LATS1 as a human suppressor gene was assessed by investigating its molecular alterations in human soft tissue sarcomas.

\section{Results}

\section{Genomic Structure of h-warts/LATS1}

As a tool that should help in the design of primers for the gene expression analysis and in the search for mutations of the $h$-warts/LATS1, we first undertook characterization of its genomic structure. After the PCR and subsequent DNA sequencing, we identified seven junctions between different exons and the $5^{\prime}-$ uncoding region within the examined sequence of the $h$-warts/LATS1 mRNA (Fig. 1, Table 1). The sizes of the exons ranged from 148 to $1514 \mathrm{bp}$. However, the 


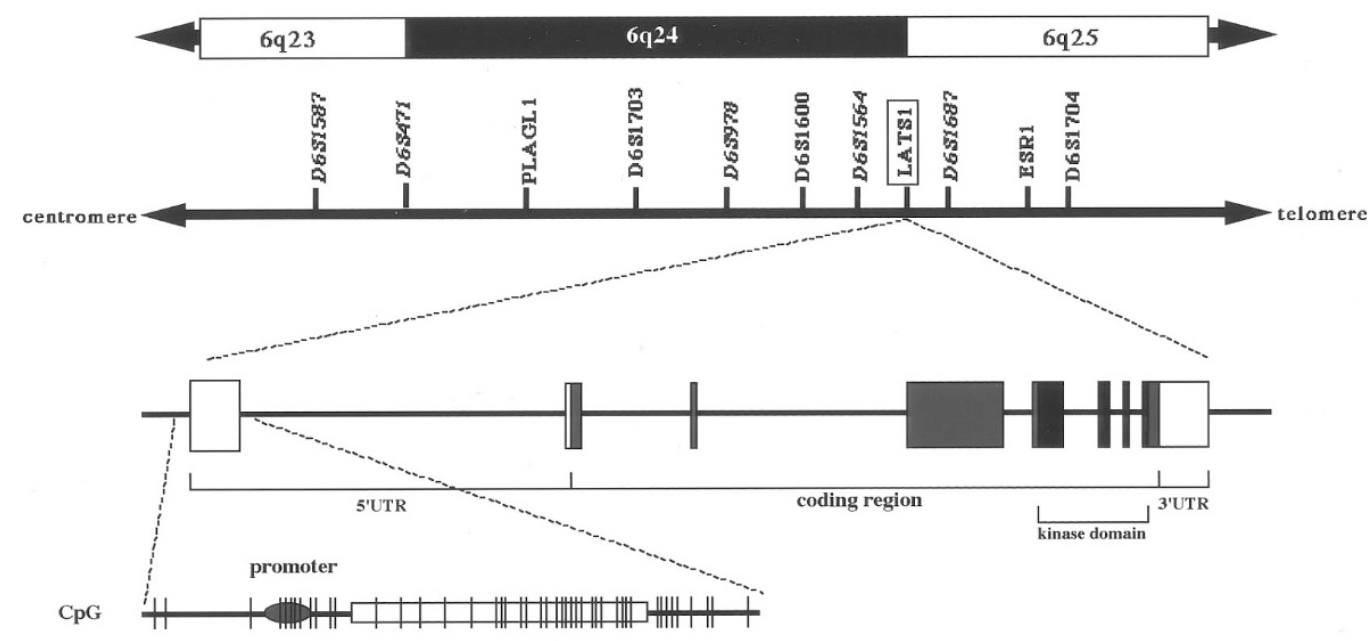

Figure 1.

Schematic representation of the $h$-warts/LATS1 and its approximate chromosomal position. Names of neighboring microsatellite markers examined in this study are italicized. Open boxes (uncoding region) and closed boxes (coding region) represent exons of the $h$-warts/LATS1. CpG dinucleotides (vertical lines) are clustered in the $5^{\prime}$ region including a putative promoter of the $h$-warts/LATS1.

Table 1. Genomic Structures of $h$-Warts/LATS1

\begin{tabular}{|c|c|c|c|c|}
\hline \multirow[b]{2}{*}{ No. } & \multirow[b]{2}{*}{$\begin{array}{l}\text { 5' Position } \\
\text { of exon }{ }^{a}\end{array}$} & \multicolumn{3}{|c|}{ Inron-exon boundaries } \\
\hline & & $\begin{array}{l}3^{\prime} \text { of the previous } \\
\text { intron }\end{array}$ & $5^{\prime}$ exon ... . 3' exon (size) & $5^{\prime}$ of the next intron \\
\hline 1 & - & .. aca gta agt agc cag & 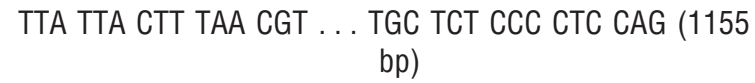 & gta ggt ccg ggg ctg.. \\
\hline 2 & 419 & $\ldots$ ttc ttt ctt tta cag & $\begin{array}{c}\text { AGT TAA TTA TTT ATA ... GCT GGA TTT GAT GAG (488 } \\
\text { bp) }\end{array}$ & gta aga atg ttt aaa ... \\
\hline 3 & 897 & $\ldots$ ttc aat tca tat cag & $\begin{array}{c}\text { GAT ATG GTT ATA CAA ... CCA GCA TGA AAC CAG }(148 \text { bp) } \\
\text {. }\end{array}$ & gtg att aat caa ata ... \\
\hline 4 & 1045 & ... ctc tta taa cca tag & $\begin{array}{c}\text { GGA ATG TGC AGC AAT ... AAT GAA ATG ATG CGG } \\
(1514 \text { bp) }\end{array}$ & gta aaa cct ttt aaa ... \\
\hline 5 & 2559 & $\ldots$ agt tta ttt gcc tag & 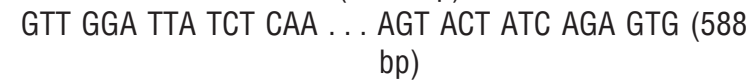 & gta agt aaa ctc ata \\
\hline 6 & 3142 & $\ldots$ tct ttt gtt gtg cag & $\begin{array}{c}\text { GTG ACC ATC CAC GGC ... TGT TGC TAC GAA CAG }(183 \\
\text { bp })\end{array}$ & gta aag cac ttc ttt \\
\hline 7 & 3325 & $\ldots$ act ttg tta ttt tag & $\begin{array}{c}\text { GAT ACA CAC AGT TGT ... GAA ACA CAA ATG AAG (107 } \\
\text { bp) }\end{array}$ & gta agg tat ggc atc... \\
\hline 8 & 3432 & $\ldots$ aaa aaa tct tta aag & GTT ATC AAC TGG CAA ... (3' boundary, not determined) & \\
\hline
\end{tabular}

structure of the remaining $3^{\prime}$ tail following the serine/ threonine kinase domain was not determined in this study. All the intronic donor and acceptor splice sites conformed to the consensus GT-AG mRNA splice sites (Table 1). The sequence coding the putative serine/ threonine kinase domain spanned four distinct exons.

\section{Gene Expression of h-warts/LATS1 in Soft Tissue Sarcomas}

In our multiplex PCR using cDNAs from the 50 tumor samples, 6 (2 leiomyosarcomas, 3 myxoid liposarcomas, and 1 malignant fibrous histiocytoma [MFH]) had no detectable gene expression of the $h$-warts/LATS1 (Fig. 2, Table 2). In addition, another leiomyosarcoma had unequivocally reduced gene expression (Fig. 2). Identical results were obtained in another PCR using a different primer set of the $h$-warts/LATS1 (Fig. 2).

\section{Analyses of h-warts/LATS1 Alterations}

To determine the molecular mechanisms of the downregulated $h$-warts/LATS1 expression in the seven sarcomas, allelic losses of 6q23-25.1 were first analyzed using five microsatellite markers for chromosome 6q23-25.1. All seven sarcomas were informative for this analysis, and an allelic loss of this chromosomal locus was identified in the leiomyosarcoma with reduced gene expression in RT-PCR (Fig. 3). 

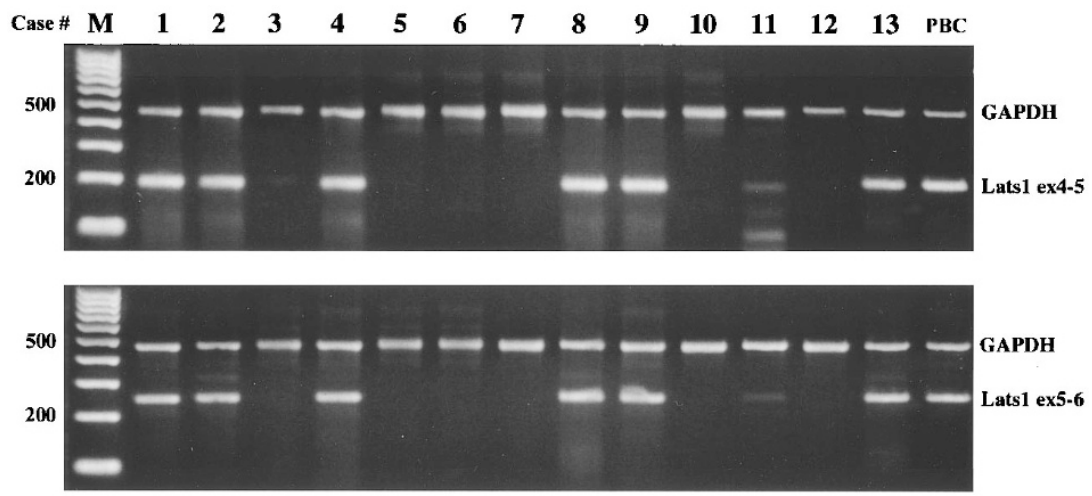

\section{Figure 2.}

Gene expressions of soft tissue sarcomas analyzed by RT-PCR for glyceraldehyde-3-phosphate dehydrogenase (GAPDH) and the sequences flanking exons 4 to 5 (upper panel) and 5 to 6 (lower panel) of the $h$-warts/LATS1. Six sarcomas (cases 3, 5, 6, 7, 10, and 12) have no detectable expression of the $h$-warts/LATS1, and one (case 11) has reduced gene expression compared with that of GAPDH. Lanes 1 to $4=$ malignant fibrous histiocytomas; lanes 5 to $8=$ myxoid liposarcomas; lanes 9 to 12 = leiomyosarcomas; lane $13=$ synovial sarcoma; $P B C=$ peripheral blood mononuclear cells, $M=100$-bp DNA ladder.

Table 2. Gene Expression of $h$-Warts/LATS1 in 50 Soft Tissue Sarcomas

\begin{tabular}{lcc}
\hline \multicolumn{1}{c}{ Tumor } & $\begin{array}{c}\text { No. of cases } \\
\text { examined }\end{array}$ & $\begin{array}{c}\text { No. of cases with } \\
\text { down-regulated gene expression }\end{array}$ \\
\hline MFH & 9 & 1 \\
Leiomyosarcoma & 7 & 3 \\
Liposarcoma, well differentiated & 6 & 0 \\
Liposarcoma, myxoid & 5 & 3 \\
Dermatofibrosarcoma protuberans & 4 & 0 \\
Extraskeletal Ewing sarcoma/PNET & 3 & 0 \\
Synovial sarcoma & 3 & 0 \\
Liposarcoma, dedifferentiated & 3 & 0 \\
Extraskeletal myxoid chondrosarcoma & 1 & 0 \\
Clear cell sarcoma & 1 & 0 \\
Epithelioid sarcoma & 1 & 0 \\
Angiosarcoma & 1 & 0 \\
Alveolar soft part sarcoma & 1 & 0 \\
Malignant peripheral nerve sheath tumor & 1 & 0 \\
Malignant hemangiopericytoma & 1 & 0 \\
Rhabdomyosarcoma, alveolar & 1 & 0 \\
Rhabdomyosarcoma, embryonal & 1 & 0 \\
Malignant mesenchymoma & 1 & 0 \\
Total & 50 & 7 \\
\hline
\end{tabular}

MFH, malignant fibrous histiocytoma; PNET, peripheral primitive neuroectodermal tumor.

In PCR-single-strand conformation polymorphism (SSCP) for gene mutations, two leiomyosarcomas showed point mutations in the coding regions of the $h$-warts/LATS1: a missense mutation at codon 566 $(\mathrm{CAA} \rightarrow \mathrm{CGA}, \mathrm{Gln} \rightarrow \mathrm{Arg})$ and a silent mutation at codon 1002 (GAT $\rightarrow$ GAC) (Fig. 4). Four sarcomas (2 myxoid liposarcomas, 1 leiomyosarcoma, and $1 \mathrm{MFH}$ ) with obvious gene expression in RT-PCR were also included in this analysis, but no gene mutation was identified. In Southern blot analysis of 4 sarcomas lacking gene expression (2 myxoid liposarcoma, 1 leiomyosarcoma, and $1 \mathrm{MFH}$ ), no aberrant bands suggesting genomic rearrangements of the $h$-warts/ LATS1 were found. No genomic amplification or deletion of the $h$-warts/LATS1 was identified either.
Although the $\mathrm{CpG}$ dinucleotide frequency in the human genome is nearly $0.6 \%$, the $5^{\prime}$ region of the $h$-warts/LATS1 has clustered CpG dinucleotides, suggesting its $5^{\prime}$ regulatory element. Using a promoter scanning program, we found a putative promoter sequence located approximately 250 to 500 bp upstream of exon 1. We therefore investigated the methylation status of this region in 13 sarcomas, including 6 sarcomas with the obvious expression of the $h$-warts/LATS1 as controls. Aberrant hypermethylation of the putative promoter of the $h$-warts/ LATS1 was identified in all but one sarcoma with reduced or no gene expression (Fig. 5). In the six sarcomas with gene expression, the promoter region was not hypermethylated. Summarized results 
Table 3. $h$-Warts/LATS1 Alterations in the Seven Sarcomas Examined

\begin{tabular}{|c|c|c|c|c|c|c|c|}
\hline \multirow[b]{2}{*}{ Tumor } & \multirow[b]{2}{*}{ Age/sex } & \multirow[b]{2}{*}{ Site } & \multicolumn{5}{|c|}{ LATS1 alterations } \\
\hline & & & Altered gene expression & $\begin{array}{l}\text { Allelic } \\
\text { loss }\end{array}$ & Gene mutation & $\begin{array}{l}\text { Genomic } \\
\text { rearrangement }\end{array}$ & $\begin{array}{l}\text { Methylated } \\
\text { 5' CpG }\end{array}$ \\
\hline $3 \mathrm{MFH}$ & $68 / \mathrm{M}$ & Thigh (It.) & + (undetectable) & - & - & - & + \\
\hline 5 Myxoid liposarcoma & $50 / \mathrm{M}$ & Thigh (rt.) & + (undetectable) & - & - & - & + \\
\hline 6 Myxoid liposarcoma & $24 / \mathrm{F}$ & Thigh (It.) & + (undetectable) & - & - & - & + \\
\hline 7 Myxoid liposarcoma & $48 / F$ & Abdominal wall & + (undetectable) & - & - & Not examined & + \\
\hline 10 Leiomyosarcoma & $46 / \mathrm{M}$ & Thigh (It.) & + (undetectable) & - & $+(\text { codon 1002) })^{a}$ & - & + \\
\hline 11 Leiomyosarcoma & $66 / F$ & Thigh (It.) & + (reduced $)$ & + & $+(\operatorname{codon} 566)^{b}$ & Not examined & - \\
\hline 12 Leiomyosarcoma & $63 / \mathrm{M}$ & Groin (rt.) & + (undetectable) & - & - & Not examined & + \\
\hline
\end{tabular}

${ }^{a}$ Silent mutation.

${ }^{b}$ Missense mutation (Gln $\rightarrow$ Arg).
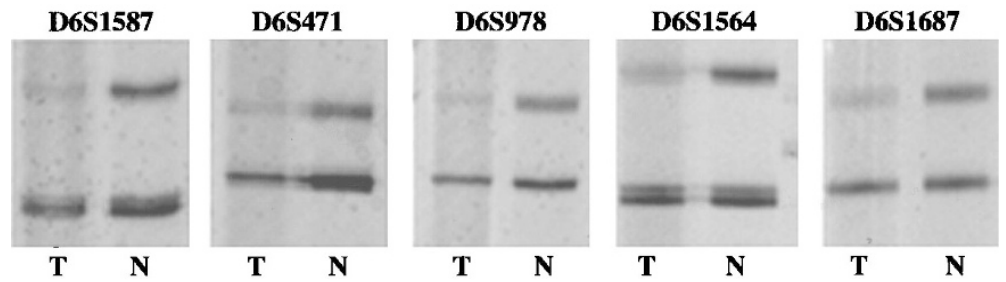

Figure 3.

An allelic loss of 6q23-25.1 in a leiomyosarcoma (case 11) demonstrated by a microsatellite marker analysis. In the tumor tissue $(T)$, loss of heterozygosity of this locus was shown by all the five markers examined. $N=$ nontumor tissue.

$\mathbf{A}$

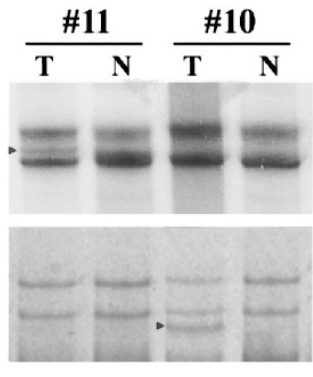

B

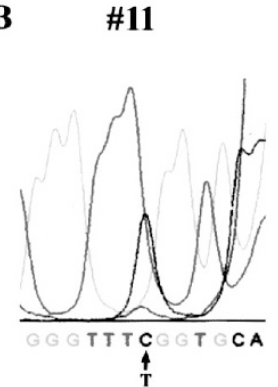

\#10

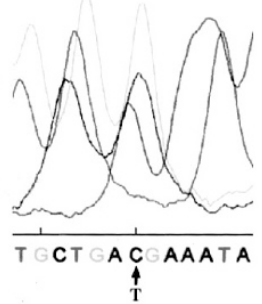

Figure 4.

A, PCR-single-strand conformation polymorphism (SSCP) for gene mutation, showing aberrant bands (arrowheads) in two leiomyosarcomas ( $T$ ) (cases 10 and 11 ). $N=$ nontumor tissue of the corresponding case; $B$, point mutations by sequencing of the PCR products extracted from the aberrant bands. An antisense cDNA sequence is shown in \#11.

of the molecular studies of the seven sarcomas examined are shown in Table 3.

\section{Discussion}

The tumor suppressor genes are considered to play a crucial role in development and/or progression of a wide variety of human neoplasms, and $p 53, R B-1$, and $p 16^{I N K 4 A} / p 14^{A R F}$ are the genes frequently investigated in human sarcomas. Abnormalities of the p53 pathway occur in nearly $50 \%$ of human carcinomas and, to a lesser extent, in sarcomas (Greenblatt et al, 1994; Latres et al, 1994; Leach et al, 1993). Aberrations in the $\mathrm{Rb}$-cyclin $\mathrm{D}$ pathway are common in certain types of sarcomas, such as leiomyosarcoma, myxoid lipo- sarcoma, and osteosarcoma (Benassi et al, 1999; Dei Tos et al, 1996, 1997). In addition, p53-deficient or p16INK4A-deficient mice frequently develop spontaneous sarcomas (Serrano et al, 1996; Williams and Jacks, 1996).

The recently identified human homolog of Drosophila tumor suppressor h-warts/LATS1 protein is localized to the mitotic apparatus and interacts with Cdc2 kinase and zyxin, a regulator of actin filament assembly during mitosis (Hirota et al, 2000; Nishiyama et al, 1999; Tao et al, 1999). h-warts/LATS1 is a member of the subfamily of protein kinases including dbf2, orb6, cot-1, ndr, and kpm, which are involved in cell cycle regulation and morphogenesis (Hori et al, 2000). Dis- 
$\mathbf{A}$

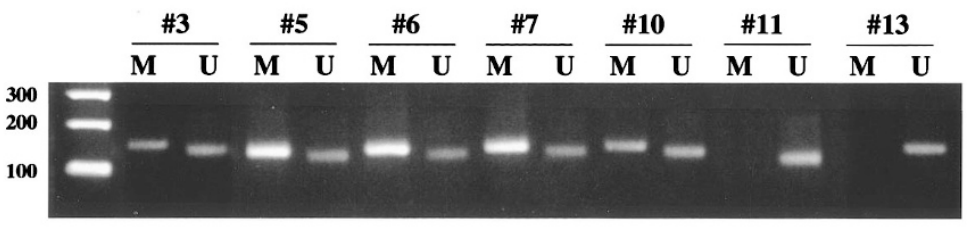

B

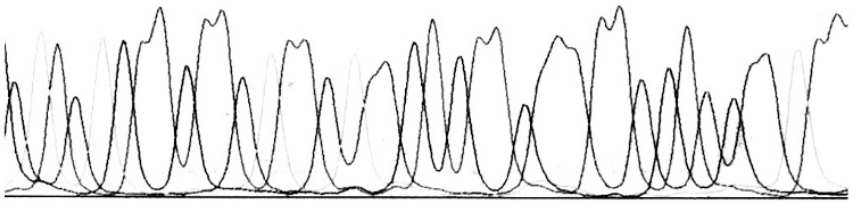

C AC TAATAACAACGCTATAATCCCAACTACTCCGAA CAA $\overline{C A T A A T A A} \overline{C A A A C A C C T A T A A T C C С А A C T A C T C ~} \overline{C A A A}$

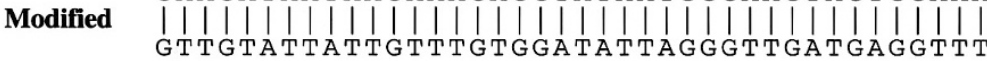
GTTGTATTATTGTTTGTGGATATTAGGGTTGATGAGGTTT
CGGCGTGGTGGGGGCGCCTGTAGTCCCAGCTACTCCGGG

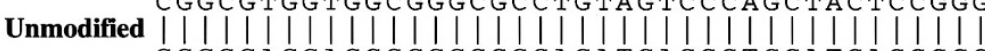
GCCGCACCACCGCCCGCGGACTCAGGGTCGATGAGCCC

Figure 5.

A, methylation-specific PCR using genomic DNA modified with bisulfite and methylated $(M)$ and unmethylated $(U)$ primers. $B$, partial sequence of the PCR product containing $\mathrm{CpG}$ dinucleotides unaffected by the chemical modification (underlined), representing methylated CpG. Modified and unmodified (original) sequences without methylated bases of the corresponding region are written.

ruption or abrogation of h-warts/LATS1 function may lead to an impaired mitotic process, potentially causing chromosomal or genetic instability, and/or dysregulation of the cell cycle by uncontrolled Cdc2/ cyclin A function. The $h$-warts/LATS1 has been shown to be ubiquitously expressed in normal fetal and adult tissues (St John et al, 1999). Therefore, the $h$-warts/ LATS1 seems to act as a human tumor suppressor gene, and development of soft tissue sarcomas and ovarian stromal tumors in mice deficient of m-LATS1 protein (Lats $1^{-/-}$) further reinforces this notion. Moreover, significant susceptibility to soft tissue sarcoma induction by carcinogens in these knockout mice has been documented (St John et al, 1999). However, phenotypic differences may be present between human and such animal models because, for example, mice heterozygous for a null mutation in the RB-1 do not develop retinoblastoma or osteosarcoma, which are the major heterozygote phenotypes in humans, but develop pituitary tumors (Jacks et al, 1992). Although recent molecular studies have shown that overexpression or ectopic expression of h-warts/ LATS1 significantly suppressed the growth of human tumor cells such as carcinoma cells and glioblastoma cells (Xia et al, 2002; Yang et al, 2001), human mesenchymal tumor cells have not been examined. Thus, we focused our study on human soft tissue sarcomas.

To date, little information regarding the status of the $h$-warts/LATS1 in clinical samples of human neoplasms is available. Only a recent molecular analysis by (Morinaga et al, 2000) has shown that no gene mutation was identified in 25 human breast cancer tissues by their RT-PCR-SSCP, and one patient had a loss of heterozygosity of the $h$-warts/LATS1. However, they did not fully address the inactivation of this gene in such a human epithelial malignancy.

When we initiated this study, the genomic structure of the $h$-warts/LATS1 had not been well characterized.
We examined intron-exon boundaries by comparing genomic DNA and cDNA sequences spanning $75 \%$ of h-LATS1 mRNA or $95 \%$ of its coding sequence recorded in GenBank. Seven junctions between different exons were identified in the genomic region analyzed, and one of them was located in its 5'-uncoding region. This genomic characterization of the $h$-warts/ LATS1 facilitated the design of primers in our RT-PCR for gene expression.

In this study, various molecular approaches were conducted to investigate the prevalence of the inactivated or down-regulated $h$-warts/LATS1 as well as their underlying mechanisms in human soft tissue sarcomas. We found that $14 \%$ of the soft tissue sarcomas examined were shown to have downregulated gene expression, suggesting a biologic role of the $h$-warts/LATS1 as a human tumor suppressor gene.

Like other tumor suppressor genes, the inactivation of the $h$-warts/LATS1 potentially involves three major mechanisms: allelic losses or deletions of the gene, gene mutations, and hypermethylation of its promoter region. In the current analysis, only one leiomyosarcoma with reduced gene expression demonstrated an allelic loss of 6q23-25.1. The low frequency of allelic alterations in our series might reflect the fact that aberrations of $6 q$ have been only rarely detected in human soft tissue sarcomas. A recent study using comparative genomic hybridization showed DNA copy number changes in $6 q$ in leiomyosarcomas, although they were mostly gains (El-Rifai et al, 1998). Interestingly, deletion of $6 \mathrm{q}$ has been demonstrated in three myxoid/round cell liposarcomas with $t(12 ; 16)$ (Altungoz et al, 1995). Because the $h$-warts/LATS1 alterations were almost confined to leiomyosarcoma and myxoid liposarcoma in our study, the molecular or genetic changes involving the $h$-warts/LATS1 may be specifically associated with these sarcoma types. 


\begin{tabular}{|c|c|c|c|}
\hline $\begin{array}{l}\text { Name of primer set } \\
\quad\left(\text { position }^{a}\right)\end{array}$ & Forward primer $\left(5^{\prime}-3^{\prime}\right)$ & $\begin{array}{l}\text { Size of PCR } \\
\text { product } \\
\text { (bp) }\end{array}$ & $\begin{array}{c}\text { Annealing } \\
\text { temperature }\left({ }^{\circ} \mathrm{C}\right)\end{array}$ \\
\hline LATS1-1 (380-534) & CTG TCC AGG AGC TCT GCT CT ATG TAG CCC ACA CGA AGG AC & 155 & 56 \\
\hline LATS1-2 (515-700) & GTC CTT CGT GTG GGC TAC AT TCA GCC TTA GCA GCA TCA GA & 186 & 56 \\
\hline LATS1-3 (681-875) & TCT GAT GCT GCT AAG GCT GA CAA GTC TTG AAG CAT TTG TGG A & 195 & 56 \\
\hline LATS1-4 (871-1051) & ACT TGC AAG CTG CTG GAT TT ACA TTC CCT GGT TTC ATG CT & 181 & 56 \\
\hline LATS1-5 (1032-1231) & AGC ATG AAA CCA GGG AAT GT GGG TGA GCT TGA ACA AAT GC & 200 & 56 \\
\hline LATS1-6 (1229-1419) & CCC TAG CAA CGG ACA GAG AG CAG GTG GGA CAG GAG AGA TT & 191 & 56 \\
\hline LATS1-7 (1402-1598) & TCT CCC GAA TCT CTC CTG TC ACC ATT CTG CAT TCC AGG TC & 197 & 56 \\
\hline LATS1-8 (1570-1746) & CAT CAG GGA GAC CTG GAA TG ATG ACG AAG GAG CAG CAG AT & 177 & 56 \\
\hline LATS1-9 (1727-1915) & ATC TGC TGC TCC TTC GTC AT GGT TGC CAT GTA GGG ATT TC & 188 & 56 \\
\hline LATS1-10 (1913-2116) & $\begin{array}{l}\text { ACC TAA CAT ACC AGT GAG GTC GGC TGT GGT ATC CAA GAA GG } \\
\text { AAA }\end{array}$ & 204 & 56 \\
\hline LATS1-11 (2103-2305) & ) TGG ATA CCA CAG CCA ATT CA CTT GGC TGA TCC TCT TTG CT & 203 & 56 \\
\hline LATS1-12 (2285-2465) & $\begin{array}{l}\text { AGC AAA GAG GAT CAG CCA AG AAA TGC TTG AGG AGA ATA ACT } \\
\text { TTG A }\end{array}$ & 181 & 56 \\
\hline $\begin{array}{l}\text { LATS } 1-13 \\
\qquad(2460-2657)\end{array}$ & $\begin{array}{l}\text { GCA TTT AAA TTC TTT ATG GAG AGA CTT GTC CAT TTT AGC CCT } \\
\text { CAA C }\end{array}$ & 198 & 56 \\
\hline $\begin{array}{l}\text { LATS1-14 } \\
\qquad(2628-2828)\end{array}$ & $\begin{array}{l}\text { CGT CTT AAA AGG GCT AAA ATG AGC TTC AGC CAG GAT ATC TCT C } \\
\text { G }\end{array}$ & 201 & 56 \\
\hline $\begin{array}{l}\text { LATS1-15 } \\
\qquad(2710-2997)\end{array}$ & $\begin{array}{l}\text { AGA TAT CCT GGC TGA AGC TGA CAA CTG CAC AGG TAA GTT CTG C } \\
\mathrm{C}\end{array}$ & 188 & 56 \\
\hline LATS1-16 (2985-3230) & $\begin{array}{l}\text { ACC TGT GCA GTT GAA AGT GTT TAA TGG CTT CAG TCT GTC TCC A } \\
\text { C }\end{array}$ & 245 & 56 \\
\hline $\begin{array}{l}\text { LATS1-17 } \\
\qquad(3209-3421)\end{array}$ & $\begin{array}{l}\text { TGG AGA CAG ACT GAA GCC ATTGTT TCT AAT GGT GTT TGT GCC A } \\
\text { A }\end{array}$ & 213 & 56 \\
\hline LATS1-18 (3401-3607) & $\begin{array}{l}\text { GGC ACA AAC ACC ATT AGA AAC CTC AGG TCA CTG GAG AAG TCA } \\
\text { A }\end{array}$ & 207 & 56 \\
\hline LATS1-M ${ }^{b}$ & 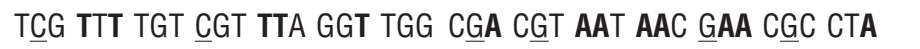 & 132 & 56 \\
\hline LATS1-U ${ }^{b}$ & 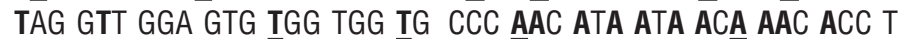 & 121 & 53 \\
\hline GAPDH (586-1037) & ACC ACA GTC CAT GCC ATC AC TCC ACC ACC CTG TTG CTG TA & 452 & 56 \\
\hline
\end{tabular}

\footnotetext{
a Position defined according to the sequences of Homo sapiens LATS homolog 1 mRNA (GenBank no. NM_004690) and human glyceraldehyde-3-phosphate dehydrogenase (GAPDH) mRNA (\#M33197).

${ }^{b}$ Sequence differences between modified primers and unmodified DNA are in boldface type, and differences between methylated/modified and unmethylated/ modified are underlined.
}

In our PCR-SSCP for gene mutations, a missense mutation was detected in one leiomyosarcoma with an allelic loss of 6q23-25.1. Although such a mutation does not seem to result in down-regulation of gene expression, the product of the mutated gene may alter its biologic property, potentially leading to the inactivation of the $h$-warts/LATS1.

Methylation of cytosine residues in $\mathrm{CpG}$ islands is an important mechanism through which genes can be differently transcribed, and abnormal methylation patterns effect either activation (hypomethylation) or silencing (hypermethylation) of genes. Methylation status of $5^{\prime}$ promoter regions of tumor suppressor genes such as the $p 16^{I N K 4 A} / p 14^{A R F}$ has been recently assessed in various human malignancies. Hypermethylation-induced inactivation of the tumor suppressor genes has been detected in variable proportions of human sarcomas; for example, the prevalence of the hypermethylated $p 16^{I N K 4 A} / p 14^{A R F}$ has varied, ranging from $0 \%$ to more than $50 \%$ in osteosarcoma (Benassi et al, 1999; Wei et al, 1999).
Because our molecular analyses for allelic losses, gene mutation, and genomic rearrangements failed to identify abnormalities potentially leading to the downregulation of the $h$-warts/LATS1 expression in six of the seven sarcomas, we then performed methylationspecific PCR of the $5^{\prime}$ putative promoter region containing $\mathrm{CpG}$ islands to verify hypermethylationinduced inactivation of the $h$-warts/LATS1 in the sarcomas. As we expected, hypermethylation of this region was identified in the six sarcomas without gene expression in RT-PCR, supporting an alternative mechanism in inactivation of the $h$-warts/LATS1. Although unmethylated sequences were also amplified by our PCR in these sarcomas, it is unknown if they were a result of incomplete hypermethylation of the $h$-warts/LATS1 or contamination of non-neoplastic tissue in the samples examined.

In conclusion, molecular abnormalities of the $h$-warts/ LATS1 in subsets of human soft tissue sarcomas suggest its functional role as a human tumor suppres- 
sor. Although clinical significance of the $h$-warts/ LATS1 remains to be determined, our findings could expand the variety of tumor-associated gene alterations involved in human mesenchymal malignancies.

\section{Materials and Methods}

Fifty cases of soft tissue sarcomas were retrieved from the files of the Department of Pathology and Oncology, School of Medicine, University of Occupational and Environmental Health, Kitakyushu, Japan. Diagnoses of the tumors were established by the combination of morphologic, immunohistochemical, and clinical features. Molecular findings such as the presence of tumor-specific fusion gene transcripts were referred to in the diagnoses of selected tumors (SYTSSX in synovial sarcoma, EWS-FL/1/ERG in the Ewing/PNET family of tumors, TLS-CHOP in myxoid liposarcoma, and TAFIl68-CHN in extraskeletal myxoid chondrosarcoma). Genomic DNA and total RNA were extracted from frozen tumor tissues and peripheral blood mononuclear cells (PBMC) provided by a healthy volunteer according to standard procedures. In a microsatellite marker analysis, DNA was isolated separately from neoplastic and non-neoplastic areas of formalin-fixed, paraffin-embedded tumor tissues by microdissection of tissue sections lightly stained with hematoxylin.

\section{PCR, RT-PCR, and DNA Sequencing}

After a treatment of DNase I (Life Technologies, Inc., Rockville, Maryland), 1 to $2 \mu \mathrm{g}$ of the extracted total RNA was reverse transcribed into cDNA using a random primer and SuperScript II reverse transcriptase (Life Technologies, Inc.) according to the manufacturer's instructions. In the molecular analyses of this study, standard PCR was performed using $0.5 \mu \mathrm{l}$ of template DNA or cDNA, $2.5 \mathrm{U}$ of Taq DNA polymerase (AmpliTaq Gold; Perkin Elmer, Norwalk, Connecticut), $1.5 \mathrm{~mm} \mathrm{MgCl} 2,10 \times$ PCR buffer (Perkin Elmer), $200 \mathrm{~mm}$ deoxynucleotide triphosphates, and $0.2 \mathrm{~mm}$ each of forward and reverse primers. A PC-801 thermal cycler (Astec Co., Shimen, Japan) was used for the PCR amplification. PCR products were cloned into a pCR2.1 vector using a TA cloning kit (Invitrogen, San Diego, California) and sequenced by an automated sequencing system (ALFexpress DNA sequencer; Pharmacia Biotech, Uppsala, Sweden).

\section{Genomic Characterization of $h$-warts/LATS1}

Based on the reported sequence of $h$-warts/LATS1 mRNA (GenBank Accession No. NM_004690), 18 sets of primers (Table 4) were designed to amplify segments ranging from 155 to $245 \mathrm{bp}$ in length and spanning most (positions 380 to 3607 ) of the mRNA sequence including a 5 '-uncoding region (positions 380 to 548 ) and the putative serine/threonine kinase domain (positions 2661 to 3575). Using the genomic DNA and CDNA of PBMC, multiple PCR was performed to find junctions between different exons under the conditions of $94^{\circ} \mathrm{C}$ for 9 minutes followed by
40 cycles at $94^{\circ} \mathrm{C}$ for 30 seconds, $56^{\circ} \mathrm{C}$ for 30 seconds, and $72^{\circ} \mathrm{C}$ for 45 seconds, and final extension at $72^{\circ} \mathrm{C}$ for 10 minutes. In the segments with no detectable bands by the standard PCR of genomic DNA, long-range PCR using $5 \mathrm{U}$ of TaKaRa LA Taq polymerase (Takara Shuzo Co., Ohtsu, Japan) was performed to more effectively amplify this region of the genomic DNA. The exact positions of the junctions between exons and sequences at the intron-exon boundaries were determined by DNA sequencing of the PCR products by referring to unpublished human DNA sequences of chromosome 6, which had been submitted directly to GenBank (Accession Nos. AL583963 and AL358852).

\section{Expression Analysis of h-warts/LATS1}

RT-PCR was performed to amplify both $h$-warts/ LATS1 and human glyceraldehyde-3-phosphate dehydrogenase (GAPDH) gene transcripts in the same microtubes. Two sets of primers (LATS1-13 and 16) (Table 4) flanking the regions including the junctions between different exons were selected to differentiate PCR products from amplified genomic DNA contamination. The PCR conditions were mostly the same as described above. In repeated preliminary RT-PCR, the number of cycles for the PCR reaction was chosen to remain within the exponential phase of the reaction and to avoid exceeding the limit of comparable intensities of the staining with ethidium bromide after gel electrophoresis. The tumors with no or unequivocally less intense bands compared with reference bands of GAPDH were regarded as down-regulated $h$-warts/ LATS1 expressions.

\section{Microsatellite Marker Analysis of 6q23-25.1}

The following five dinucleotide $(\mathrm{CA})$ repeat markers, listed in order from centromere to telomere, were analyzed by PCR: D6S1587, D6S471, D6S978, D6S1564, and D6S1687. The sequences of these satellite markers are distributed within a region of approximately $16 \mathrm{Mbp}$ in length at chromosome 6q2325.1 , to which the $h$-warts/LATS1 is localized (Nishiyama et al, 1999). The sequences of the PCR primers for these markers are available from the Genome Database (http://www.gdb.org). Forty cycles for the PCR reaction were used in this assay. The PCR products were loaded onto polyacrylamide gels and then stained with silver (Bio-Rad, Hercules, California).

\section{Mutation Analysis of $h$-warts/LATS1}

To screen gene mutations in most of the coding sequence of the $h$-warts/LATS1, SSCP was analyzed after the PCR using genomic DNA or CDNA with the above 18 sets of primers. The PCR products were heat denatured and loaded onto polyacrylamide gels at $15^{\circ} \mathrm{C}$ for 90 minutes with a peltier temperatureregulated electrophoresis unit (Genephor; Pharmacia Biotech). The gels were visualized with silver, and aberrant bands were removed from the gels. Gene mutations were further confirmed by DNA sequencing 
of the extracted PCR products from the aberrant bands. DNA with the same alteration in different clones was considered to be mutated.

\section{Southern Blot Analysis for Gene Rearrangement}

Ten micrograms of high molecular weight DNA of tumor tissues were digested with $100 \mathrm{U}$ of restriction enzymes (EcoRI, HindIII, HaellI) and loaded onto 1\% agarose gels. After treatment with $0.25 \mathrm{M} \mathrm{HCl}$, the digested DNA was transferred to positively charged nylon membranes (Amersham Pharmacia Biotech, Tokyo, Japan) and hybridized with an alkaline phosphatase-labeled probe for $h$-warts/LATS1 cDNA flanking the serine/threonine kinase domain (980 bp) that was generated by PCR. Detection of hybridized signals was made by an enhanced chemiluminescence detection system (AlkPhos Direct; Amersham Pharmacia Biotech).

\section{Methylation Analysis of CpG Islands in h-warts/LATS1 Promoter}

To identify a putative promoter sequence in the $5^{\prime}$ region of the $h$-warts/LATS1, a genomic sequence (approximately $2 \mathrm{~kb}$ in length) including a part of exon 1 was analyzed by a computer-based online program (PROSCAN Ver 1.7, http://bimas.dcrt.nih.gov/molbio) (Prestridge, 1995). The methylation status of CpG dinucleotides (islands) in the $5^{\prime}$ putative promoter region of the $h$-warts/LATS1 was assessed by methylation-specific PCR using primer sets for methylated and unmethylated sequences of genomic DNA modified with bisulfite (CpGenome DNA Modification Kit; Invitrogen, Purchase, New York) (Herman et al, 1996). Sequences of the primers used are shown in Table 4. The modified DNA was amplified by the PCR under the conditions of 40 cycles of $94^{\circ} \mathrm{C}$ for 15 seconds, $56^{\circ} \mathrm{C}$ (methylated) or $53^{\circ} \mathrm{C}$ (unmethylated) for 15 seconds, and $72^{\circ} \mathrm{C}$ for 30 seconds. Sequences of the PCR products were confirmed by DNA sequencing.

\section{Acknowledgements}

The authors thank Drs. K. Yokoyama (National Kokura Hospital) and M. Ushijima (Kitakyushu Municipal Hospital) for providing tumor tissues.

\section{References}

Altungoz O, Meloni AM, Peier A, Zalupski M, Spanier S, Brooks JS, and Sandberg AA (1995). Deletion 6q in three cases of mixed-type liposarcoma in addition to $t(12 ; 16)(q 13$; p11). Cancer Genet Cytogenet 79:104-110.

Benassi MS, Molendini L, Gamberi G, Ragazzini P, Sollazzo MR, Merli M, Asp J, Magagnoli G, Balladelli A, Bertoni F, and Picci $P$ (1999). Alteration of $\mathrm{pRb} / \mathrm{p} 16 / \mathrm{cdk} 4$ regulation in human osteosarcoma. Int J Cancer 84:489-493.

Dei Tos AP, Maestro R, Doglioni C, Piccinin S, Libera DD, Boiocchi M, and Fletcher CD (1996). Tumor suppressor genes and related molecules in leiomyosarcoma. Am J Pathol 148:1037-1045.
Dei Tos AP, Piccinin S, Doglioni C, Vukosavljevic T, Mentzel T, Boiocchi M, and Fletcher CD (1997). Molecular aberrations of the G1-S checkpoint in myxoid and round cell liposarcoma. Am J Pathol 151:1531-1539.

El-Rifai W, Sarlomo-Rikala M, Knuutila S, and Miettinen M (1998). DNA copy number changes in development and progression in leiomyosarcomas of soft tissue. Am J Pathol 153:985-990.

Greenblatt MS, Bennett WP, Hollstein M, and Harris CC (1994). Mutations in the p53 tumor suppressor gene: Clues to cancer etiology and molecular pathogenesis. Cancer Res 54:4855-4878.

Herman JG, Graff JR, Myöhänen S, Nelkin BD, and Baylin SB (1996). Methylation-specific PCR: A novel PCR assay for methylation status of $\mathrm{CpG}$ islands. Proc Natl Acad Sci USA 93:9821-9826.

Hirota T, Morisaki T, Nishiyama Y, Marumoto T, Tada K, Hara T, Masuko N, Inagaki M, Hatakeyama K, and Saya H (2000). Zyxin, a regulator of actin filament assembly, targets the mitotic apparatus by interacting with h-warts/LATS1 tumor suppressor. J Cell Biol 149:1073-1086.

Hooper M (1998). Tumour suppressor gene mutations in humans and mice: Parallels and contrasts. EMBO J 17:67836789.

Hori T, Takaori-Kondo A, Kamikubo Y, and Uchiyama T (2000). Molecular cloning of a novel human protein kinase, $\mathrm{kpm}$, that is homologous to warts/lats, a Drosophila tumor suppressor. Oncogene 19:3101-3109.

Jacks T, Fazeli A, Schmitt EM, Bronson RT, Goodell MA, and Weinberg RA (1992). Effects of an Rb mutation in the mouse. Nature 359:295-300.

Kinzler KW and Vogelstein B (1998). Familial cancer syndromes: The role of caretakers and gatekeepers. In: Vogelstein B and Kinzler KW, editors. The genetic basis of human cancer. New York: McGraw-Hill, 241-242.

Latres E, Drobnjak M, Pollack D, Oliva MR, Ramos M, Karpeh M, Woodruff JM, and Cordon-Cardo C (1994). Chromosome 17 abnormalities and TP53 mutations in adult soft tissue sarcomas. Am J Pathol 145:345-355.

Leach FS, Tokino T, Meltzer P, Burrell M, Oliner JD, Smith S, Hill DE, Sidransky D, Kinzler KW, and Vogelstein B (1993). p53 Mutation and MDM2 amplification in human soft tissue sarcomas. Cancer Res 53:2231-2234.

Morinaga N, Shitara Y, Yanagita Y, Koida T, Kimura M, Asao T, Kimijima I, Takenoshita S, Hirota T, Saya H, and Kuwano $\mathrm{H}$ (2000). Molecular analysis of the $h$-warts/LATS1 gene in human breast cancer. Int J Oncol 17:1125-1129.

Nishiyama Y, Hirota T, Morisaki T, Hara T, Marumoto T, lida S, Makino K, Yamamoto H, Hiraoka T, Kitamura N, and Saya H (1999). A human homolog of Drosophila warts tumor suppressor, h-warts, localized to mitotic apparatus and specifically phosphorylated during mitosis. FEBS Lett 459:159165.

Prestridge DS (1995). Predicting Pol II promoter sequences using transcription factor binding sites. J Mol Biol 249:923932.

Serrano M, Lee H, Chin L, Cordon-Cardo C, Beach D, and DePinho RA (1996). Role of the INK4a locus in tumor suppression and cell mortality. Cell 85:27-37. 
St John MA, Tao W, Fei X, Fukumoto R, Carcangiu ML, Brownstein DG, Parlow AF, McGrath J, and Xu T (1999). Mice deficient of Lats1 develop soft-tissue sarcomas, ovarian tumours and pituitary dysfunction. Nat Genet 21:182-186.

Tao W, Zhang S, Turenchalk GS, Stewart RA, St John MA, Chen W, and Xu T (1999). Human homologue of the Drosophila melanogaster lats tumour suppressor modulates CDC2 activity. Nat Genet 21:177-181.

Wei G, Lonardo F, Ueda T, Kim T, Huvos AG, Healey JH, and Ladanyi M (1999). CDK4 gene amplification in osteosarcoma: Reciprocal relationship with INK4A gene alterations and mapping of 12q13 amplicons. Int J Cancer 80:199-204.
Williams BO and Jacks T (1996). Mechanisms of carcinogenesis and the mutant mouse. Curr Opin Genet Dev 6:65-70.

Xia H, Qi H, Li Y, Pei J, Barton J, Blackstad M, Xu T, and Tao W (2002). LATS1 tumor suppressor regulates G2/M transition and apoptosis. Oncogene 21:1233-1241.

Yang X, Li D-M, Chen W, and Xu T (2001). Human homologue of Drosophila lats, LATS1, negatively regulates growth by inducing G2/M arrest or apoptosis. Oncogene 20:65166523. 